\title{
A novel approach to assessing bisphenol-A hazards using an in vitro model system
}

\author{
Md Saidur Rahman ${ }^{\dagger}$, Woo-Sung Kwon ${ }^{\dagger}$, Sung-Jae Yoon, Yoo-Jin Park, Buom-Yong Ryu and Myung-Geol Pang ${ }^{*}$
}

\begin{abstract}
Background: Although the toxicological impacts of the xenoestrogen bisphenol-A (BPA) have been studied extensively, but the mechanism of action is poorly understood. Eventually, no standard method exists for evaluating the possible health hazards of BPA exposure. Considering mice spermatozoa as a potential in vitro model, we investigated the effects of BPA exposure $(0.0001,0.01,1$, and $100 \mu \mathrm{M}$ for $6 \mathrm{~h})$ on spermatozoa and the related mechanisms of action. The same doses were also employed to evaluate protein profiles of spermatozoa as a means to monitor their functional affiliation to diseases.

Results: Our results demonstrated that high concentrations of BPA negatively affect sperm motility, viability, mitochondrial functions, and intracellular ATP levels by activating the mitogen-activated protein kinase, phosphatidylinositol 3-kinase, and protein kinase-A pathways. Moreover, short-term exposure of spermatozoa to high concentrations of BPA induced differential expressions of 24 proteins. These effects appeared to be caused by protein degradation and phosphorylation in spermatozoa. Proteins differentially expressed in spermatozoa from BPA treatment groups are putatively involved in the pathogenesis of several diseases, mainly cancer, carcinoma, neoplasm, and infertility.

Conclusions: Based on these results, we propose that BPA adversely affects sperm function by the activation of several kinase pathways in spermatozoa. In addition, BPA-induced changes in the sperm proteome might be partly responsible for the observed effects in spermatozoa, subsequently involve in the pathogenesis of many diseases. Therefore, we anticipated that current strategy might broadly consider for the health hazards assessment of other toxicological agents.
\end{abstract}

Keywords: Bisphenol-A, Health hazards, In-vitro, Spermatozoa, Proteomics, Bioinformatics databases, Diseases, Signaling pathways

\section{Background}

Bisphenol-A (BPA) is an estrogenic endocrine disruptor and is produced annually in large quantities for the production of polycarbonate plastics and epoxy resins. Because of hormone-like properties, concerns have been raised about the sustainability of BPA in consumer products. The Centers for Disease Control and Prevention has identified high levels of urinary BPA in $>90 \%$ of the U.S. population [1]. BPA is associated with impaired reproductive functions as well as other diseases, such as cancer, diabetes, obesity, cardiovascular disease, thyroid dysfunction, developmental disorders, and miscarriages

\footnotetext{
*Correspondence: mgpang@cau.ac.kr

${ }^{\dagger}$ Equal contributors

Department of Animal Science and Technology, Chung-Ang University, Anseong, Gyeonggi-Do 456-756, Republic of Korea
}

[2]. Several in vivo studies have reported that BPAexposed males are prone to reproductive developmental disorders $[3,4]$ and that the spermatozoa of exposed males are incapable of proper sperm-oocyte binding, thereby affecting embryonic growth [5]. Recently we have reported that BPA-induced modifications of fertilityrelated proteins in spermatozoa are associated with increased reproductive health risk [6].

Genomic and non-genomic pathways are two proposed mechanisms by which xenoestrogens exert their hormone-like action. In the genomic pathway, xenoestrogens trigger estrogen receptors (ERs, nuclear and cytoplasmic) and lead to the transcription of target genes, thereby affecting cell function [7]. In the non-genomic pathway, $G$ protein-coupled receptors (GPCRs) together with membrane-bound ERs 
(non-genomic) cause rapid estrogenic signaling through activation of mitogen-activated protein kinase (MAPK) and phosphatidylinositol 3-kinase (PI3K), changes in cAMP/protein kinase-C (PKC), and fluctuation of intracellular calcium [8]. Consistently, BPA also has been reported to exert its effects via activation of MAPK and PI3K in human ovarian cancer cells $[9,10]$. As demonstrated, mature spermatozoa contain functional GPCRs [11] and non-genomic ERs [12], therefore, provide a valid model to investigate the molecular mechanism of BPA action [6, 13, 14].

Recent advances in proteomic research have offered a new vista for developing proteomic biomarkers of chemical exposure, with the aim of evaluating hostile health effects [15]. In particular, two-dimensional gel electrophoresis (2-DE) coupled with mass spectrometry (MS) has been used effectively in several toxicology studies and in high-throughput industrial applications. A direct comparison of protein expression levels from control versus altered conditions reveals a set of biomarkers representative of the altered state. Since spermatozoa have no functional protein synthesizing machinery, therefore provide a valid model for proteomic analysis [16-18]. Consequently, several laboratories have applied this technique to develop diagnostic biomarkers of spermatozoa in different functional states $[16,17]$. However, to date, efforts to establish protein expression profiles indicative of xenoestrogen (BPA) exposure in spermatozoa have not been attempted. Therefore, the objectives of the current study were: 1) to determine whether different concentrations of BPA could affect sperm functions, and if so, to investigate the mechanisms linked with these observed effects and 2) to identify the differentially expressed proteins of exposure as a manner to screen their functional affiliation to diseases.

\section{Results}

The effect of BPA on motility, viability, and intracellular lactate dehydrogenase (LDH) levels in spermatozoa

The percentage of motile spermatozoa decreased significantly in the presence of $100 \mu \mathrm{M}$ of BPA $(p<0.05)$ (Fig. 1a). However, the sperm viability was affected significantly by exposure to both 1 and $100 \mu \mathrm{M}$ BPA $(p<$ 0.05) (Fig. 1b). In contrast, a non-significant increase of
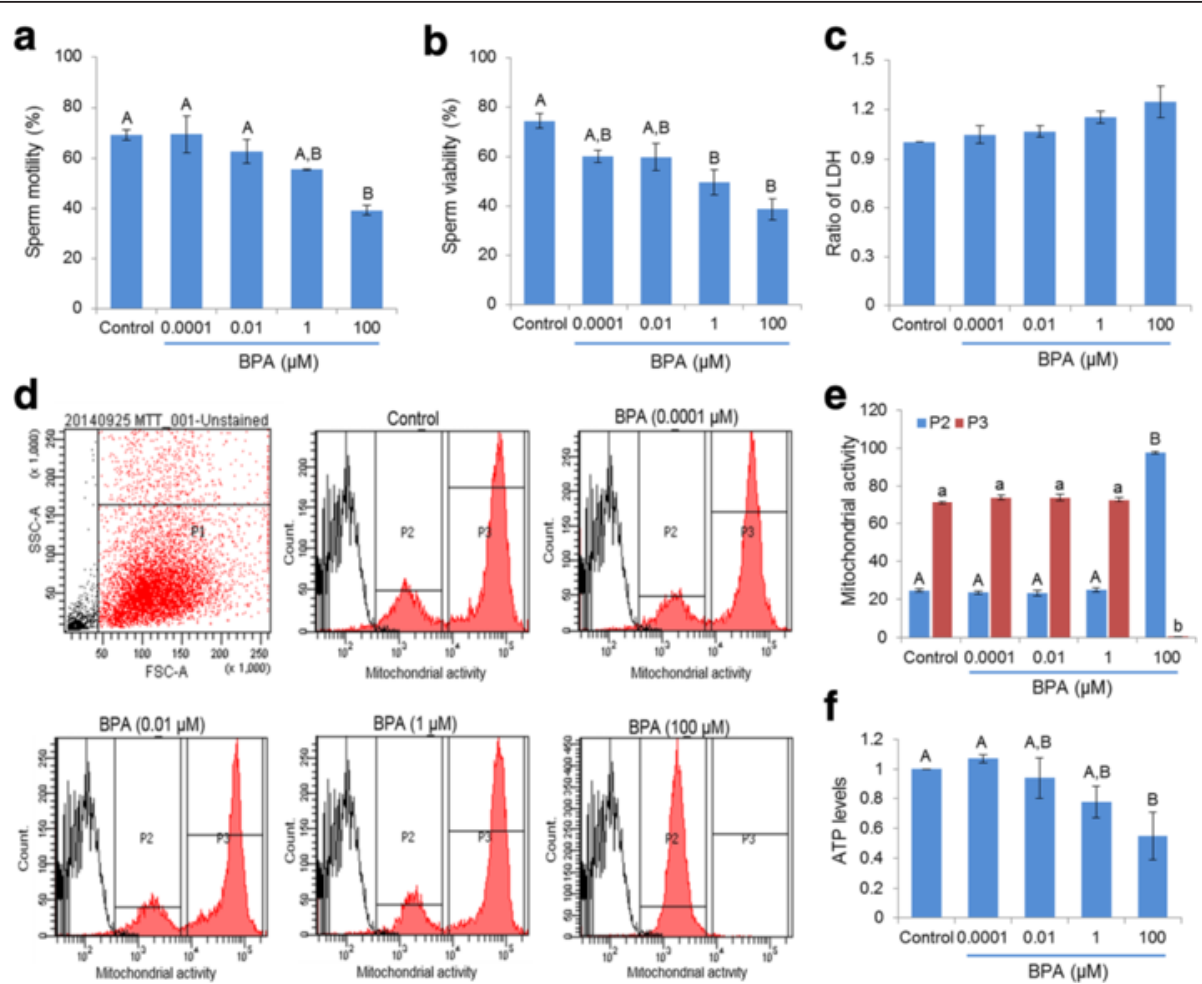

Fig. 1 Effect of BPA on motility, viability, lactate dehydrogenase (LDH), mitochondrial activity, and intracellular ATP ([ATP]) levels. (a) Differences in motility in BPA-treated and control spermatozoa. (b) Differences in number of viable spermatozoa between the control and BPA treatment. (c) Differences in LDH levels in control and BPA-treated spermatozoa. (d) Representative flow cytometry images of mitochondrial activity in control and BPA-treated spermatozoa. (e) The bar represents the difference in mitochondrial activity detected by flow cytometry in control and BPA-treated spermatozoa. (f) Differences in [ATP]; levels between control and BPA-treated spermatozoa. Data are presented as mean \pm SEM ( 4 replicates). Values with different superscript characters $(A, B, a, b)$ indicate significant differences between the control and treatment groups as determined by one-way ANOVA $(p<0.05)$ 
intracellular LDH levels was noted in all concentrations of BPA tested (Fig. 1c).

\section{The effect of BPA on mitochondrial activity and} intracellular ATP $\left([A T P]_{i}\right)$ levels in spermatozoa

Mitochondrial respiration produces ATP, which is required to maintain sperm motility and other biochemical modifications required for fertilization [19]. Current study showed that a high concentration of BPA $(100 \mu \mathrm{M})$ significantly altered the mitochondrial activity of spermatozoa $(p<0.05)$ (Fig. $1 \mathrm{~d}$ and e). To validate whether these alterations of mitochondrial activity were correlated with overall $[\mathrm{ATP}]_{\mathrm{i}}$ production, we measured the $[\mathrm{ATP}]_{\mathrm{i}}$ levels in spermatozoa. Our results revealed that high concentrations of BPA were also able to significantly reduce $[\mathrm{ATP}]_{\mathrm{i}}$ levels compared with those of other tested concentrations and the control $(p<0.05)$ (Fig. 1f).

\section{BPA stimulates MAPK, PI3K, and PKA pathways in spermatozoa}

As reported previously $[9,10]$, BPA activates the MAPK and PI3K signaling pathways in ovarian cancer cells. To evaluate this possibility in spermatozoa, activation of MAPK(p38) and PI3K(p85) were evaluated using western blot analyses. Our results showed that BPA induced a dose-dependent increase in both phospho-MAPK (p38) and phospho-PI3K (p85) (Fig. 2a-d). Further, it has been demonstrated that the activation of the a

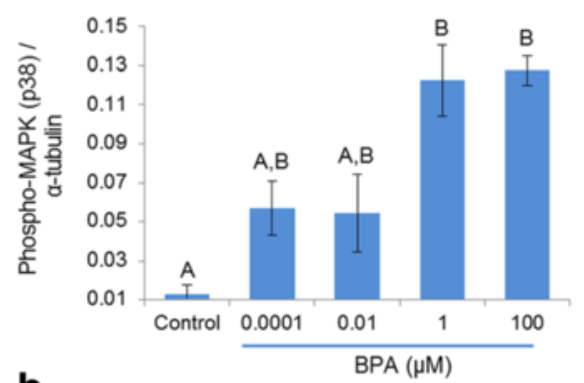

b

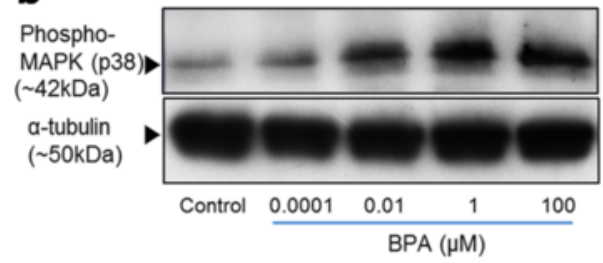

e

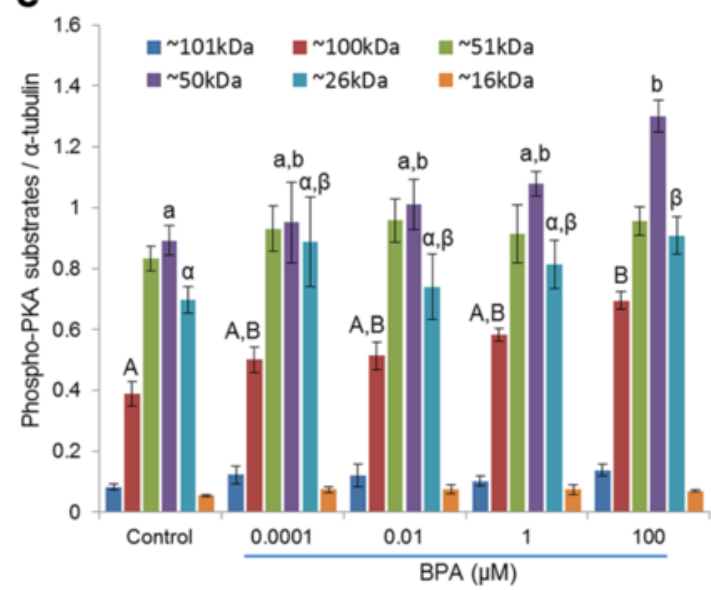

C

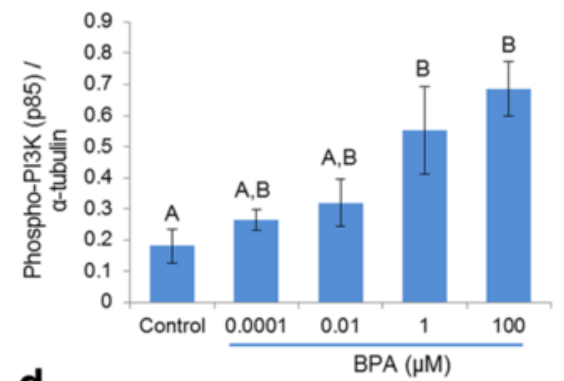

d

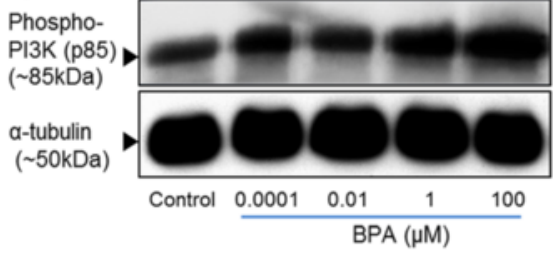

f

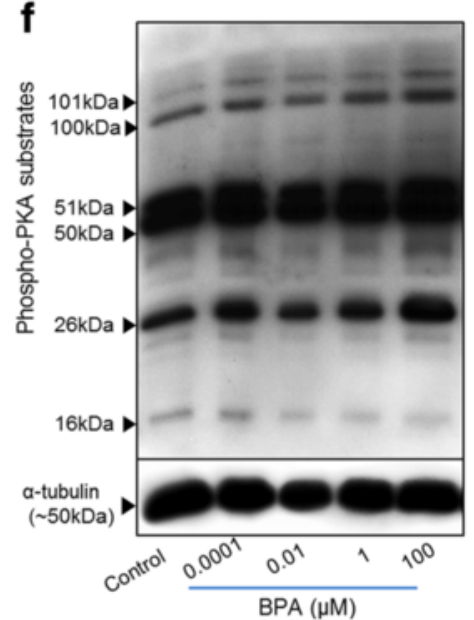

Fig. 2 Effect of BPA on MAPK (p38), PI3K (p85), and PKA signalings pathways in spermatozoa. (a) The bar represents the densities of phosphoMAPK (p38) in BPA-treated and control spermatozoa. (b) Representative western blot image of phospho-MAPK (p38) probed with specific antibody. (c) Densities of phospho-PI3K (p85) in BPA-treated and control spermatozoa. (d) Representative western blot image of phospho-PI3K (p85) probed with specific antibody. (e) Densities of PKA substrate species in BPA-treated and control spermatozoa. (f) Phospho-PKA substrates were probed with an anti-phospho-PKA antibody. Lane 1: control; lane 2: $0.0001 \mu \mathrm{M}$ BPA; lane 3: $0.01 \mu \mathrm{M}$ BPA; lane 4: $1 \mu \mathrm{M}$ BPA; and lane 5: $100 \mu \mathrm{M}$ BPA. Data are presented as mean \pm SEM (3 replicates). Values with different superscript characters $(A, B, a, b, a, a, \beta)$ indicate significant differences between the control and treatment groups as determined by one-way ANOVA $(p<0.05)$ 
MAPK/PI3K signaling pathways regulates PKA activity to maintain complex cross-talk between these pathways $[20,21]$. Therefore, to further discriminate the PKA activity in spermatozoa due to exposure of BPA, PKA levels were also measured. Significant increases in the levels of three PKA substrates $(\sim 100,50$, and $26 \mathrm{kDa})$ were observed in the presence of $100 \mu \mathrm{M}$ of BPA $(p<0.05)$ (Fig. 2e and f).

\section{Identification and quantification of proteins differentially expressed in control and BPA-exposed spermatozoa}

To learn pathophysiological importance of BPA exposure, we applied a proteomic approach (2-DE coupled with MS) for identification of differentially expressed proteins in spermatozoa. On average, 399 spots were consistently detected in all gels, 250 of which shared almost similar expression patterns across treatments. Fifty spots showed a dose-dependent expression profile, but significant $(P<$ 0.05 ) changes were noticed in 29 spots. In our assessment of the dose-dependent expression profiles, we avoided nine spots that demonstrated non-linear differences in expression between treated and control groups. Finally, we identified 24 spots by electrospray ionization tandem mass spectrometry (ESI-MS/MS). The identified proteins spots and their normalized spot values in the control and BPAtreated spermatozoa are shown in Fig. 3 and Table 1, respectively (the detailed information regarding peptide sequence, matches peptides, and used searching engine of identified proteins were provided in Additional file 1: Table S1). Our results showed that 24 proteins (16 downand 9 up-regulated) were differentially expressed between BPA-treated $(100 \mu \mathrm{M})$ and control spermatozoa (Table 1). The number of differentially expressed proteins in control vs $0.01 \mu \mathrm{M}$ and control $v s 1 \mu \mathrm{M}$ were 3 (2 down- and 1 up-regulated) and 7 (4 down- and 3 up-regulated), respectively (Table 1). Additionally, using Gene Ontology annotation in bioinformatics databases (UniProt-GOA and Human Sperm Proteome), the identified proteins were characterized according to their biological function as listed in Table 1.

\section{Functional analysis and signaling pathways of differentially expressed proteins}

Pathway Studio program was used to identify signaling pathways regulated by the differentially expressed proteins. Our results showed a significant association between five canonical signaling pathways and some of the differentially expressed proteins $(p<0.05)$ (Table 2). We also identified and schematized the clinical significance of these proteins using a MedScan Reader (v5.0) search and Pathway Studio, respectively. The disease processes regulated by the differentially expressed proteins are compiled in Fig. 4a.

\section{Western blot analysis of six representative proteins and phospho-tyrosine proteins}

To validate the 2-DE results, six differentially expressed proteins, such as glyceraldehyde-3-phosphate dehydrogenase (GAPDH), cytochrome b-c1 complex (UQCRFS1), peroxi redoxin-5 (PRDX5), glutathione peroxidase (GPX4), actin, cytoplasmic 1 (ACTB), and glutathione S-transferase (GSTM5) were further verified by western blot analyses using their commercially available antibodies. The western blot results confirmed the differentially expressed protein levels visualized in 2-DE (Additional file 2: Figure S1). Western blot also was applied to investigate the phospho-tyrosine levels (PY20) in both the treated and control spermatozoa. Significant increases in the levels of two tyrosine-phosphorylated species $(\sim 100$ and $\sim 24 \mathrm{kDa})$ were detected in BPA-treated ( 1 and $100 \mu \mathrm{M})$ spermatozoa compared with the control $(p<0.05)$ (Additional file 2: Figure S2).

\section{Discussion}

BPA has been regarded as a ubiquitous environmental chemical to which we are exposed in everyday life. Considering the toxicological importance of BPA, here we attempted to investigate whether several concentrations of BPA would affect sperm functions and to investigate the molecular mechanisms. Additionally, BPA was employed to separate differentially expressed proteins in spermatozoa as a manner to monitor their functional affiliation to several disease processes.

Our results showed that higher concentrations of BPA significantly decreased sperm motility (Fig. 1a) and viability (Fig. 1b), even the doses that were potentially nontoxic (Fig. 1c). Several earlier studies also demonstrated the similar action of BPA on sperm motility in rats and mice $[6,22,23]$. It has been reported that mitochondrial respiration mainly provides ATP to support sperm motility [19]. To evaluate this possibility, the effect of $\mathrm{BPA}$ on mitochondrial activity and $[\mathrm{ATP}]_{\mathrm{i}}$ also were evaluated. Consistent with the motility and viability data, $100 \mu \mathrm{M}$ BPA altered the mitochondrial activity significantly (Fig. 1d and e) and decreased the $[\mathrm{ATP}]_{\mathrm{I}}$ (Fig. 1f). Collectively, our findings support that BPA $(\sim 100 \mu \mathrm{M})$ alters the mitochondrial activity and decreases $[\mathrm{ATP}]_{\mathrm{i}}$ and, thus, could affect sperm functions (motility/viability).

BPA has been demonstrated to activate the MAPK and PI3K signaling pathways in cancer cells [9, 10, 24]. It has been demonstrated that p38 (MAPK) and p85 (PI3K) proteins are rapidly tyrosine-phosphorylated in response to extracellular stimuli and trigger subsequent activation of the kinase systems $[25,26]$. We have shown that the addition of BPA to spermatozoa leads to a significant dose-dependent induction of the phospho-MAPK (p38) and phospho-PI3K (p85), thereby activating both 


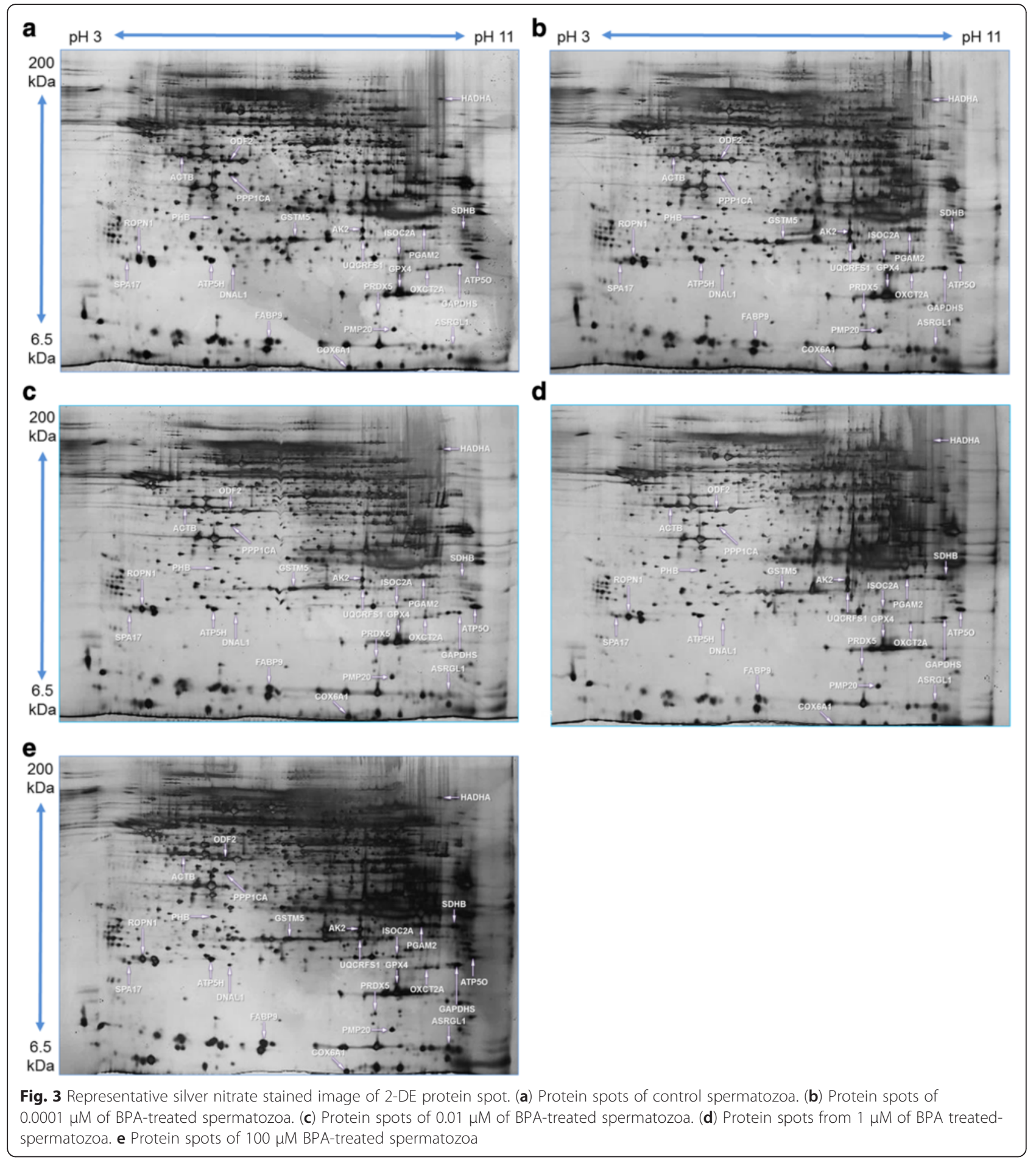

kinase pathways (Fig. 2a-d). Activation of MAPK [27] and PI3K [28] also has been reported in ejaculated bovine spermatozoa due to exposure of extracellular stimuli. On the other hand, PKA has been shown to be essential for the regulation of major signaling pathways in spermatozoa [6, 29], as well as cross-talk between MAPK and PI3K signaling $[20,21]$. Further, the activation of the PKA pathway by structurally diverse estrogenic compounds, including BPA, also has been reported [24]. Therefore, we performed western blot analysis for PKA activity (Fig. 2e and f), in treated and control spermatozoa. Consistent with previous finding in other cells, collectively our results support that BPA activates the MAPK, PI3K, and PKA 
Table 1 Proteins with a significantly lower or higher expression in treatment and control groups

\begin{tabular}{|c|c|c|c|c|c|c|c|c|}
\hline \multirow[t]{2}{*}{ Symbol } & \multirow[t]{2}{*}{ Protein description } & \multirow[t]{2}{*}{ gi no. } & \multirow{2}{*}{$\begin{array}{l}\text { MASCOT } \\
\text { score }^{*}\end{array}$} & \multicolumn{5}{|c|}{ Relative intensity $^{a}$ (normalized) } \\
\hline & & & & Control & 0.0001 & 0.01 & 1 & 100 \\
\hline \multicolumn{9}{|c|}{ Energy metabolism (46 \%) } \\
\hline ATP5H & ATP synthase subunit $d$, mitochondrial & gi|21313679 & 58 & $1^{\mathrm{A}}$ & $0.99 \pm 0.15^{\mathrm{A}}$ & $0.93 \pm 0.12^{\mathrm{A}}$ & $0.63 \pm 0.01^{A, B}$ & $0.36 \pm 0.12^{B}$ \\
\hline ATP5O & ATP synthase subunit $O$, mitochondrial & gi|20070412 & 69 & $1^{\mathrm{A}}$ & $0.97 \pm 0.19^{A}$ & $0.68 \pm 0.14^{\mathrm{A}, \mathrm{B}}$ & $0.62 \pm 0.08^{A, B}$ & $0.44 \pm 0.07^{\mathrm{B}}$ \\
\hline HADHA & $\begin{array}{l}\text { Trifunctional enzyme subunit alpha, } \\
\text { mitochondrial }\end{array}$ & gi|33859811 & 66 & $1^{\mathrm{A}}$ & $0.71 \pm 0.01^{A, B}$ & $0.41 \pm 0.03^{B, C}$ & $0.40 \pm 0.04^{B, C}$ & $0.31 \pm 0.10^{c}$ \\
\hline COX6A1 & $\begin{array}{l}\text { Cytochrome c oxidase subunit } 6 \mathrm{~A} 1 \text {, } \\
\text { mitochondrial }\end{array}$ & gi|1352173 & 49 & $1^{\mathrm{A}}$ & $0.68 \pm 0.19^{A, B}$ & $0.28 \pm 0.07^{B, C}$ & $0.23 \pm 0.06^{B, C}$ & $0.17 \pm 0.01^{c}$ \\
\hline PGAM2 & Phosphoglycerate mutase 2 & gi|9256624 & 53 & $1^{\mathrm{A}}$ & $0.99 \pm 0.13^{\mathrm{A}}$ & $0.76 \pm 0.13^{A, B}$ & $0.72 \pm 0.08^{A, B}$ & $0.34 \pm 0.07^{\mathrm{B}}$ \\
\hline OXCT2A & $\begin{array}{l}\text { Succinyl-CoA:3-ketoacid coenzyme A } \\
\text { transferase } 2 A \text {, mitochondrial }\end{array}$ & gi|81881929 & 36 & $1^{\mathrm{A}}$ & $0.71 \pm 0.16^{\mathrm{A}, \mathrm{B}}$ & $0.67 \pm 0.07^{A, B}$ & $0.65 \pm 0.04^{A, B}$ & $0.43 \pm 0.09^{B}$ \\
\hline ISOC2A & $\begin{array}{l}\text { Isochorismatase domain-containing } \\
\text { protein } 2 \mathrm{~A} \text {, mitochondrial }\end{array}$ & gi|197333728 & 73 & $1^{\mathrm{A}}$ & $0.83 \pm 0.19^{A, B}$ & $0.79 \pm 0.08^{A, B}$ & $0.75 \pm 0.10^{A, B}$ & $0.34 \pm 0.12^{B}$ \\
\hline GAPDH & $\begin{array}{l}\text { Glyceraldehyde-3-phosphate } \\
\text { dehydrogenase, testis-specific }\end{array}$ & gi|2494630 & 37 & $1^{\mathrm{A}}$ & $1.09 \pm 0.14^{\mathrm{A}}$ & $1.11 \pm 0.25^{A, B}$ & $1.12 \pm 0.31^{A, B}$ & $2.17 \pm 0.28^{B}$ \\
\hline AK2 & Adenylate kinase 2, mitochondrial isoform & gi|34328230 & 70 & $1^{\mathrm{A}}$ & $1.13 \pm 0.18^{A, B}$ & $1.19 \pm 0.27^{\mathrm{A}, \mathrm{B}}$ & $1.37 \pm 0.11^{\mathrm{A}, \mathrm{B}}$ & $2.05 \pm 0.14^{B}$ \\
\hline SDHB & Succinate dehydrogenase Ip subunit & gi|34328286 & 63 & $1^{\mathrm{A}}$ & $2.59 \pm 0.66^{\mathrm{A}, \mathrm{B}}$ & $3.02 \pm 0.29^{\mathrm{A}, \mathrm{B}}$ & $3.32 \pm 0.56^{\mathrm{B}}$ & $3.27 \pm 0.38^{B}$ \\
\hline UQCRFS1 & $\begin{array}{l}\text { Cytochrome b-c1 complex subunit } \\
\text { Rieske, mitochondrial }\end{array}$ & gi|13385168 & 62 & $1^{\mathrm{A}}$ & $1.56 \pm 0.17^{A, B}$ & $1.62 \pm 0.40^{A, B}$ & $1.72 \pm 0.32^{\mathrm{A}, \mathrm{B}}$ & $2.16 \pm 0.01^{B}$ \\
\hline
\end{tabular}

Cytoskeletal/structural proteins (25\%)

$\begin{array}{lllllllll}\text { ROPN1 } & \text { Ropporin-1 } & \text { gi|74227586 } & 202 & 1^{A} & 0.88 \pm 0.12^{\mathrm{A}} & 0.78 \pm 0.10^{\mathrm{A}, \mathrm{B}} & 0.71 \pm 0.10^{\mathrm{A}, \mathrm{B}} & 0.49 \pm 0.01^{\mathrm{B}} \\ \text { ACTB } & \text { Actin, cytoplasmic 1 } & \text { gi|6671509 } & 51 & 1^{\mathrm{A}} & 0.93 \pm 0.17^{\mathrm{A}} & 0.92 \pm 0.16^{\mathrm{A}} & 0.49 \pm 0.04^{\mathrm{B}} & 0.47 \pm 0.04^{\mathrm{B}} \\ \text { FABP9 } & \text { Fatty acid-binding protein 9 } & \text { gi|166897974 } & 68 & 1^{\mathrm{A}} & 0.94 \pm 0.05^{\mathrm{A}} & 0.86 \pm 0.13^{\mathrm{A}} & 0.68 \pm 0.03^{\mathrm{A}, \mathrm{B}} & 0.47 \pm 0.07^{\mathrm{B}} \\ \text { ODF2 } & \text { Outer dense fiber protein 2 } & \text { gi|2290719 } & 57 & 1^{\mathrm{A}} & 0.68 \pm 0.14^{\mathrm{A}, \mathrm{B}} & 0.62 \pm 0.19^{\mathrm{A}, \mathrm{B}} & 0.59 \pm 0.16^{\mathrm{A}, \mathrm{B}} & 0.13 \pm 0.01^{\mathrm{B}} \\ \text { PMP20 } & \text { Peroxisomal membrane protein 20 } & \text { gi|6746357 } & 79 & 1^{\mathrm{A}} & 1.06 \pm 0.05^{\mathrm{A}} & 1.10 \pm 0.06^{\mathrm{A}, \mathrm{B}} & 1.17 \pm 0.22^{\mathrm{A}, \mathrm{B}} & 2.11 \pm 0.01^{\mathrm{B}} \\ \text { ASRGL1 } & \text { Isoaspartyl peptidase/L-asparaginase } & \text { gi|81875980 } & 52 & 1^{\mathrm{A}} & 1.38 \pm 0.12^{\mathrm{A}, \mathrm{B}} & 2.09 \pm 0.87^{\mathrm{A}, \mathrm{B}} & 2.79 \pm 0.42^{\mathrm{A}, \mathrm{B}} & 3.23 \pm 0.36^{\mathrm{B}}\end{array}$

Fertility Related Proteins (17\%)

\begin{tabular}{|c|c|c|c|c|c|c|c|c|}
\hline PHB & Prohibitin & gi|6679299 & 68 & $1^{\mathrm{A}}$ & $0.91 \pm 0.12^{\mathrm{A}}$ & $0.81 \pm 0.20^{\mathrm{A}, \mathrm{B}}$ & $0.75 \pm 0.12^{A, B}$ & $0.43 \pm 0.05^{\mathrm{B}}$ \\
\hline PPP1CA & $\begin{array}{l}\text { Serine/threonine-protein phosphatase } \\
\text { PP1-alpha catalytic subunit }\end{array}$ & gi|49065812 & 50 & $1^{\mathrm{A}}$ & $0.94 \pm 0.16^{\mathrm{A}}$ & $0.60 \pm 0.20^{\mathrm{A}, \mathrm{B}}$ & $0.47 \pm 0.07^{A, B}$ & $0.36 \pm 0.05^{\mathrm{B}}$ \\
\hline DNAL1 & Dynein light chain 1 , axonemal & gi|164607162 & 67 & $1^{\mathrm{A}}$ & $0.74 \pm 0.01^{A, B}$ & $0.65 \pm 0.14^{\mathrm{A}, \mathrm{B}}$ & $0.64 \pm 0.23^{A, B}$ & $0.49 \pm 0.13^{B}$ \\
\hline SPA17 & Sperm surface protein Sp17 & gi|6755614 & 58 & $1^{\mathrm{A}}$ & $1.11 \pm 0.21^{\mathrm{A}}$ & $1.39 \pm 0.81^{\mathrm{A}}$ & $1.59 \pm 0.05^{\mathrm{A}, \mathrm{B}}$ & $2.08 \pm 0.17^{B}$ \\
\hline \multicolumn{9}{|c|}{ tress response proteins/ROS metabolism (12 \%) } \\
\hline GSTM5 & Glutathione S-transferase Mu 5 & gi|6754086 & 70 & $1^{\mathrm{A}}$ & $0.92 \pm 0.26^{\mathrm{A}, \mathrm{B}}$ & $0.67 \pm 0.08^{\mathrm{A}, \mathrm{B}}$ & $0.48 \pm 0.09^{B}$ & $0.32 \pm 0.08^{B}$ \\
\hline GPX4 & Glutathione peroxidase & gi|3075477 & 65 & $1^{\mathrm{A}}$ & $1.71 \pm 0.08^{A, B}$ & $1.87 \pm 0.19^{\mathrm{B}}$ & $2.24 \pm 0.24^{B}$ & $2.25 \pm 0.15^{B}$ \\
\hline PRDX5 & Peroxiredoxin-5, mitochondrial & gi|6755114 & 73 & $1^{\mathrm{A}}$ & $1.28 \pm 0.17^{A, B}$ & $1.30 \pm 0.15^{A, B}$ & $2.02 \pm 0.11^{B}$ & $2.35 \pm 0.09^{B}$ \\
\hline
\end{tabular}

*MASCOT score is $-10 \log (\mathrm{P})$, where $\mathrm{P}$ is the probability that the observed match is a random event. Individual scores $>30$ indicate identity or extensive homology $(p<0.05)$. ${ }^{a}$ Relative spots intensity in control and BPA-treated $(\mu \mathrm{M})$ spermatozoa. Data are presented as mean \pm SEM $(3$ replicates). Values with different superscript characters $(A, B, C)$ indicate significant differences between the control and treatment groups as determined by one-way ANOVA $(P<0.05)$

signaling in spermatozoa and ultimately affects the cell functions.

A recent technological advance in proteomics is that researchers have successfully developed diagnostic biomarkers of several diseases [15]. Nevertheless, complete proteomic biomarkers indicative of xenoestrogen exposure (eg BPA) in spermatozoa still remain unknown. Therefore, we have performed 2-DE coupled with MS to identify unique protein expression profiles in BPA-treated spermatozoa to predict the pathophysiological consequences of exposure. Our results showed that 24 proteins (16 down- and 9 up-regulated) 
Table 2 Signaling pathways associated with differentially expressed proteins as identified by Pathway Studio program

\begin{tabular}{lll}
\hline Signaling Pathways & Overlapping Entities & $P$-value \\
\hline Respiratory chain and oxidative phosphorylation & UQCRFS1, SDHB, COX6A1, ATP5O & $<0.001$ \\
Glutathione metabolism & GSTM5, GPX4, PRDX5 & 0.017 \\
Notch Pathway & ACTB, PPP1CA, HADHA, PGAM2, ODF1, GAPDH & 0.017 \\
EphrinR - > actin signaling & ACTB, ODF1 & 0.030 \\
Adipocytokine Signaling & SDHB, HADHA, PGAM2, GAPDH & 0.031
\end{tabular}

BPA-induced differentially expressed proteins were entered into the Pathway Studio program to identify the significantly signaling pathways. The probabilities of the signaling pathways were determined using the Fisher's exact test $(p<0.05)$

were dose dependently $(p<0.05)$ expressed between BPA-treated and control spermatozoa (Table 1). Protein degradation (down-regulation) in spermatozoa due to environmental factors is well studied [6, 16, 17]; however, the manner in which protein levels are increased in vitro need to be investigated. Mature spermatozoa are silent in both transcription and translation or are poorly capable of translation [6]. Therefore, protein up-regulation could be achieved by posttranslational modification, such as tyrosine phosphorylation $[6,16,17,30]$. A similar increase in protein tyrosine phosphorylation was also found in spermatozoa due to BPA exposure (Additional file 2: Figure S2). Taken together with several recent studies $[6,16,17,30]$, our results suggest that exposure to BPA induces protein degradation and phosphorylation in spermatozoa, ultimately affecting sperm function.

A total of 11 (46\%) proteins involved in energy metabolism exhibited altered levels in BPA-exposed $(100 \mu \mathrm{M})$ spermatozoa compared with those of the control (Table 1). Proteins warranting attention for being degraded were mitochondrial ATP synthase subunit d (ATP5H), mitochondrial ATP synthase subunit O (ATP5O), mitochondrial trifunctional enzyme subunit alpha (HADHA), mitochondrial cytochrome c oxidase subunit 6A1 (COX6A1), phosphoglycerate mutase 2 (PGAM2), mitochondrial succinyl-CoA:3-ketoacid coenzyme A transferase 2A (OXCT2A), and mitochondrial isochorismatase domain-containing protein $2 \mathrm{~A}$ (ISOC2A) (Table 1). Most of the degraded proteins

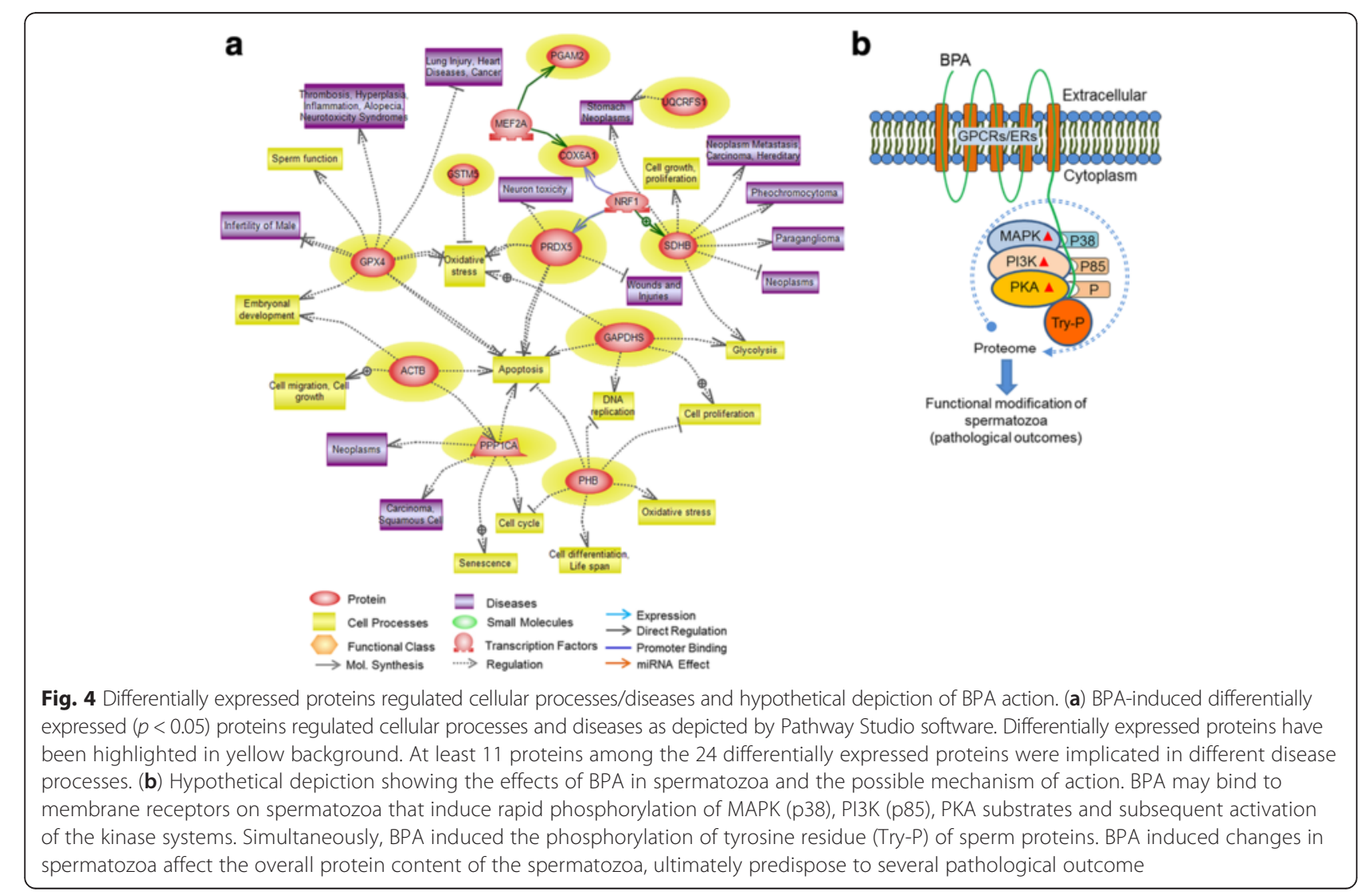


identified in this group were mitochondrial isoforms, thus predominantly involved in energy metabolism. Therefore, down-regulation of these proteins could affect the overall mitochondrial functions and ATP production as also demonstrated in current study (Fig. 1f). Clinically, the ATP synthase subunit and HADHA have been shown to be down-regulated in type 2 diabetes [31] and hepatocellular cancer cells [32], respectively. Moreover, COX6A1 exerts a protective role against ROS-induced cell damage; thus, its down-regulation could lead to abnormal cell functions [33]. It is important to note that a significant downregulation of HADHA and COX6A1 were detected between the control and $0.01 / 1 \mu \mathrm{M}$ BPA exposure (Table 1), and therefore, these proteins could provide potential biomarkers of low dose effects. On the other hand, the up-regulated proteins of energy metabolism group were GAPDH, mitochondrial isoform of adenylate kinase 2 (AK2), succinate dehydrogenase Ip subunit (SDHB), and UQCRFS1 (Table 1). It is unclear, how sperm motility (Fig. 1a) and ATP (Fig. 1f) are decreased in the condition when energy metabolism proteins are increased. However, the level of expression may be suboptimal to overcome BPA-mediated toxicity, and/or the substantial increases may result in atypical functioning of these proteins.

Six cytoskeletal/structural proteins $(25 \%)$ were found to be significantly altered in $100 \mu \mathrm{M}$ BPA-treated spermatozoa compared to those in the control cells (Table 1). The down-regulated proteins were ropporin-1 (ROPN1), ACTB, fatty acid-binding protein 9 (FABP9), and outer dense fiber protein 2 (ODF2) (Table 1). The cytoskeletal/ structural proteins are important for cytoplasmic integrity, cell movement, and signal transduction maintenance. Therefore, down-regulation of these proteins could affect the cellular physiology. Clinically, down-regulation of these proteins has been found in non-motile, abnormal headed, infertile spermatozoa [18, 34]. Additionally, the functional affiliation of these proteins also has been reported to prostatic carcinoma and metastatic melanoma of the testis $[35,36]$. In contrast, the upregulated proteins were peroxisomal membrane protein 20 (PMP20) and isoaspartyl peptidase/L-asparaginase (ASRGL1) (Table 1); interestingly, neither of them had been previously described in spermatozoa. Therefore, they might be the possible biomarkers of BPA hazard assessment; however, further study needs to be conducted to strengthen this hypothesis.

Additionally, in fertility-related proteins (17\%), sperm surface protein Sp17 (SPA17) was increased by BPA exposure $(100 \mu \mathrm{M})$, whereas prohibitin (PHB), serine/ threonine-protein phosphatase PP1-alpha catalytic subunit (PPP1CA), and axonemal dynein light chain 1 (DNAL1) were decreased (Table 1). SPA17 has been reported to play a potential role in sperm-egg interactions; however, increased functionality of this protein induced immature acrosome reaction and tumor changes in cells [37], which might support our current findings. In contrast, the degraded proteins are reported to play potential roles in the regulation of sperm motility and fertilization [38, 39]. Thus, degradation of these proteins results in spermatozoa with reduced motility and viability (Fig. 1a and b). Lastly, among stress response proteins (12\%), GPX4 and PRDX5 were up-regulated, and GSTM5 was down-regulated by BPA (Table 1). It has been reported that toxic levels of ROS in mammalian spermatozoa are strongly associated with male infertility [29]. Thus, failure of the antioxidant defense system of spermatozoa to remove excess ROS levels ultimately compromises normal sperm function and fertilization. Therefore, the elevated levels of GPX4 and PRDX5 observed in the present study were assumed to offer the sperm cells a survival advantage (Fig. 1c) in a BPAcontaining microenvironment. In addition, significantly increased expression of GPX4 was noted with $0.01 \mu \mathrm{M}$ BPA treatment compared with control (Table 1), and thus, GPX4 could represent a brilliant candidate biomarker of low-dose exposure.

Our data showed that 13 of 24 differentially expressed proteins in BPA-treated spermatozoa were significantly $(p<0.05)$ correlated with five canonical pathways (Table 2). Since normal functioning of these pathways regulates cell physiology, manipulation of pathway entities definitely correlates with pathological consequences/diseases. Using Pathway Studio program, we show that the disease processes regulated by the altered proteins of these pathways were putatively involved in cancer, carcinoma, neoplasm, infertility, and so, (Fig. 4a). However, the Pathway Studio program showed the particular proteins regulated pathways, cellular processes, and diseases from entries in the PubMed and other related databases; thus, the proteins that exhibited a non-significant relationship with several pathways might also have potential clinical significance.

\section{Conclusions}

Here, we describe the in vitro effects of BPA on mouse spermatozoa and the possible mechanisms of action. Additionally, we identified 24 differentially expressed proteins in spermatozoa indicative of BPA exposure and showed their affiliation with several diseases (Fig. 4b). These predicted protein markers could be used as potential candidates for developing a novel theranostic strategy for the management of BPA toxicity. Additionally, we anticipated that the proteomics and informatics tools used current study might consider for the health hazard assessment of other toxicological agents. 
However, further studies are required to validate preliminary finding and to better elucidate the possible clinical applications of these techniques.

\section{Methods}

\section{Ethical approval}

All procedures with animals were performed in accordance to protocols approved by the Institutional Animal Care and Use Committee of Chung-Ang University, Seoul, Republic of Korea.

\section{Media and chemicals}

All chemicals and reagents were purchased from SigmaAldrich (St. Louis, MO, USA), unless otherwise stated. Modified Tyrode's medium (osmolality $300 \pm 20 \mathrm{mOsm} /$ $\mathrm{kg}, \mathrm{pH} 7.2 \pm 0.2$ ) was prepared freshly and used as basic medium (BM) $[6,29]$. The BM was pre-incubated 1 day prior to the experiment and supplemented with bovine serum albumin (BSA; $4 \mathrm{mg} / \mathrm{mL}$ ). BPA was dissolved in dimethylsulfoxide (DMSO) and added to a final concentration to treat the spermatozoa. The control spermatozoa were treated with DMSO only. An estrogenic positive control was not considered in the present study because BPA has been reported to act via other signaling pathways together with ERs; thus, it is not obvious whether BPA and natural estrogens have similar effects [40, 41].

\section{BPA dose selection}

The exposure scheme consisted of four different doses of BPA: $0.0001,0.01,1$, and $100 \mu \mathrm{M}$. The doses up to $1 \mu \mathrm{M}$ were comparable to the acceptable human daily exposure levels [22]. It has been demonstrated that low dose of BPA $(\sim 0.01 \mu \mathrm{M})$ advances early embryonic development, whereas a comparatively higher dose $(\sim 100 \mu \mathrm{M})$ decreases the development rate of the embryo [42, 43]. More recently, genotoxic and mitogenic effects of BPA has been demonstrated at the dose of $0.01-0.1 \mu \mathrm{M}$, in mammary cells [44]. Therefore, range of concentrations $(0.001-100 \mu \mathrm{M})$ was considered in current study to clarify in vitro adverse effect levels of BPA in spermatozoa.

\section{Collection and preparation of spermatozoa and their exposure to BPA}

Spermatozoa were collected from sexually mature ICR male mice (Nara Biotech, Seoul, Korea) following published procedures $[6,29]$. Briefly, both cauda epididymides from each mouse were collected and the associated fat was removed. The epididymides were then placed in cell culture dishes with BM containing $0.4 \%$ BSA, and punctured using a sterile needle to release spermatozoa. The released spermatozoa were incubated for approximately $10 \mathrm{~min}$ with $5 \% \mathrm{CO}_{2}$ in air at $37{ }^{\circ} \mathrm{C}$ to facilitate dispersal. An initial experiment was designed to determine the potential of BPA to alter a) sperm motility and b) viability. For this, the spermatozoa were incubated $\left(5 \% \mathrm{CO}_{2}\right.$ in air at $\left.37{ }^{\circ} \mathrm{C}\right)$ with $100 \mu \mathrm{M} \mathrm{BPA}$ for different periods of time (ranging from 0.5 to $8 \mathrm{~h}$ at 30min intervals) to investigate the potential shift of both parameters. Finally, $6 \mathrm{~h}$ was identified as the minimum effective period of BPA exposure. Therefore, the sperm suspension was incubated for $6 \mathrm{~h}$ under the same conditions in $B M$ supplemented with various concentrations of BPA.

\section{Assessment of sperm motility}

Sperm motility was evaluated using computer-assisted sperm analysis (CASA) (SAIS plus version 10.1; Medical Supply, Seoul, Korea) according to described methods $[6,29]$. The $10 \times$ phase contrast objective was used by the SAIS software to relay and analyze the spermatozoa. Five fields of each sample were randomly selected to evaluate the movement of at least 250 sperm.

\section{Hypo-osmotic swelling test (HOST)}

To evaluate sperm viability and functional integrity (membrane), we used the hypo-osmotic swelling test (HOST) as described previously [45]. The sperm swelling patterns were classified broadly as viable and/or nonviable according to the WHO 2010 manual and by using the Microphot-FXA microscope (Nikon).

\section{Detection of LDH}

To determine cytotoxicity, the CytoTox $96^{\circ}$ assay kit (Promega, Fitchburg, WI, USA), which is based on the colorimetric detection of $\mathrm{LDH}$, was used according to the method described previously [6, 29]. The LDH activity was measured as the absorbance at $490 \mathrm{~nm}$ by using a luminometer (GEMINI EM, Molecular Devices Corporation) and was calculated using the SoftMax Pro 5 software. Activity is reported as the ratio of the fluorescence of BPA-treated samples to that of the control.

\section{Detection of mitochondrial activity}

Mitochondrial membrane potential, defined as mitochondrial activity, was measured by rhodamine 123 (R123) staining according to the manufacturer's directions and the described method [45]. The samples were analyzed by flow cytometry (Becton Dickinson, Franklin Lakes, NJ, USA) with an excitation wavelength of $488 \mathrm{~nm}$ and an emission wavelength of $525 \mathrm{~nm}$. Ten thousand cells in each sample were considered to obtain R123 signal, and the signal was analyzed using the CellQuest software (Becton Dickinson). Mitochondrial activity was reported as the fluorescence of the BPA-treated samples compared with that of the control. 


\section{Detection of $[A T P]_{i}$ levels}

$[\mathrm{ATP}]_{\mathrm{i}}$ was detected using an ATP Bioluminescence Assay Kit (CLS II; Roche Molecular Biochemicals, Mannheim, Germany) according to the described method [29]. The luminescence signal was detected using a Microplate Multimode Reader (GloMax ${ }^{-}$-Multi; Promega, Madison, WI, USA). The $[A T P]_{i}$ is reported as the ATP (signal) of the BPA-treated samples compared with that of the control.

\section{Preparation of spermatozoa for proteomic experiments}

The BPA-treated and control spermatozoa after $6 \mathrm{~h}$ of incubation were washed twice by centrifugation $(100 \times g$ for $2.5 \mathrm{~min}$ ) at room temperature (RT), re-suspended in $\mathrm{BM}$, and allowed to swim-up at $37^{\circ} \mathrm{C}$ for an additional $15 \mathrm{~min}$. Swim-up was performed to separate the motile sperm fraction from immature spermatozoa and somatic cells [46-48]. The motile sperm fraction was then carefully collected, and the samples were checked for the nonappearance of immature spermatozoa and somatic cells using light microscopy and Hoechst staining (Additional file 2: Figure S3).

\section{2-DE and gel-image analysis}

To extract proteins from the spermatozoa, $50 \times 10^{6}$ cells were incubated in rehydration buffer for $1 \mathrm{~h}$ at $4{ }^{\circ} \mathrm{C}[17$, 18, 48]. Then, $250 \mu \mathrm{g}$ aliquots of sperm protein were placed in $450 \mu \mathrm{L}$ of rehydration buffer in a rehydration tray with $24 \mathrm{~cm}$-long NL Immobiline DryStrips ( $\mathrm{pH} 3-$ 11; Amersham, Piscataway, NJ, USA) for $12 \mathrm{~h}$ at $4{ }^{\circ} \mathrm{C}$. The first-dimension electrophoresis (1-DE) was performed using an IPGphor IEF apparatus, and the strips were focused according to previously described settings [17, 18, 48]. After iso-electrofocusing, the strips were equilibrated for $15 \mathrm{~min}$ at RT by using equilibration buffer $\mathrm{A}$, whereas the second equilibration was performed using equilibration buffer B [17, 18, 48]. Afterward, 2DE was carried out on $12.5 \%(\mathrm{w} / \mathrm{v})$ SDS-PAGE gels with the strips at $100 \mathrm{~V}$ for $1 \mathrm{~h}$ and then at $500 \mathrm{~V}$ until the bromophenol blue front began to migrate off the gels. The gels were silver-stained for image analysis according to the manufacturer's instructions (Amersham, Piscataway, NJ, USA). The silver-stain was used to conduct the present study because this method is highly sensitive, capable of detecting minimal variants of protein in the gel, and is compatible with downstream processing, such as mass spectrometry [18]. The gels were scanned using a high-resolution GS-800 calibrated scanner (Bio-Rad, Hercules, CA, USA). Detected spots were matched and analyzed by comparing the gels from spermatozoa treated with BPA and the control using PDQuest 8.0 software (Bio-Rad, Hercules, CA, USA). Finally, the spot density was calculated and normalized as the ratio of the spot on the BPA-treated (spermatozoa) gel to that on the control gel.

\section{Protein identification In-gel digestion}

Proteins were subjected to in-gel trypsin digestion according to the protocol established previously $[17,18]$. Excised gel spots were destained with $100 \mu \mathrm{L}$ of destain solution $(30 \mathrm{mM}$ potassium ferricyanide, $100 \mathrm{mM}$ sodium thiosulfate) by shaking for $5 \mathrm{~min}$. Then, the gel spots were incubated with $200 \mathrm{mM}$ ammonium bicarbonate for $20 \mathrm{~min}$. The gel pieces were dehydrated with $100 \mu \mathrm{L}$ of acetonitrile and dried in a vacuum centrifuge. The above procedure was repeated thrice. Then, the dried gel pieces were rehydrated with $20 \mu \mathrm{L}$ of $50 \mathrm{mM}$ ammonium bicarbonate containing $0.2 \mu \mathrm{g}$ of modified trypsin for 45 min on ice. Seventy microliters of $50 \mathrm{mM}$ ammonium bicarbonate was added after removal of solution. Digestion was performed overnight at $37{ }^{\circ} \mathrm{C}$. The peptide solution was desalted using a C18 nano column (homemade, Waters Corp, Milford, MA, USA).

\section{Desalting and concentration}

The custom-made chromatographic columns were used for desalting and concentration of the peptide mixture. A column consisting of $100-300 \mathrm{~nL}$ of Poros reverse phase R2 material (PerSeptive Biosystems, Framingham, MA, USA) was packed in a constricted GELoader tip (Eppendorf, Hamburg, Germany). The liquid was forced gently into the column using a $10-\mathrm{mL}$ syringe. Thirty microliters of the peptide mixture from the digestion supernatant was diluted with $30 \mu \mathrm{L}$ of $5 \%$ formic acid, loaded onto the column, and washed with $30 \mu \mathrm{L}$ of $5 \%$ formic acid. Peptides were eluted with $1.5 \mu \mathrm{L}$ of $50 \%$ methanol/49 \% H2O/1 \% formic acid directly into a precoated borosilicate nanoelectrospray needle (New Objective, Woburn, MA, USA) for analysis by tandem mass spectrometry (MS/MS).

\section{ESI-MS/MS}

MS/MS of peptides generated by in-gel digestion was performed by nano-ESI on a MicroQ-TOF III mass spectrometer (Bruker Daltonics, Germany) at RT. A potential of $1 \mathrm{kV}$ was applied to the precoated borosilicate nanoelectrospray needles (EconoTipTM, New Objective) in the ion source and combined with a nitrogen backpressure of $0-5$ psi to produce a stable flow rate (10$30 \mathrm{~nL} / \mathrm{min}$ ). The cone voltage was $800 \mathrm{~V}$. The quadrupole analyzer was used to select precursor ions for fragmentation in the hexapole collision cell. Product ions were analyzed using an orthogonal TOF analyzer, fitted with a reflector, a micro-channel plate detector, and a time-to-digital converter. The data were processed using a peptide sequence system. 


\section{Database search}

An MS/MS ion search was allocated as the ion search preference in the MASCOT software (Matrix 20 Science, Boston, MA, USA). Peptide fragment files were obtained from the peptide peaks in ESI-MS by ESI-MS/MS. Trypsin was selected as the enzyme with one potentially missed cleavage site. ESI-QTOF was selected as the instrument type. The peptide fragments were searched based on the database using the MASCOT (v2.4, Matrix Science) and FASTA search engine, and the search was limited to Mus musculus taxonomy in NCBInr, UniprotKB/TrEMBL and UniprotKB/Swissprot database. The mass tolerance was set at \pm 1 and $\pm 0.6 \mathrm{Da}$ for the peptides and fragments, respectively. High-scores were defined as those above the default significance threshold in MASCOT $(p<0.05$, peptide score, $>30)$.

\section{Western blotting}

Western blot analysis was performed as previously described $[6,29]$. The antibodies were diluted in $3 \%$ blocking agent: anti-phospho-p38 MAPK Antibody (1:1000, Cell Signaling, Danvers, MA), Anti-PI3K p85 (phosphor Y607) antibody (1:1000, Abcam, Cambridge, UK), antiphospho-PKA substrate antibody (1:10,000; Cell Signaling Technology, MA, USA), anti-phosphotyrosine antibody (PY20, 1:2,500, Abcam), anti- GAPDH antibody (1:1000, Abcam), anti-UQCRFS1 antibody (1:15,000, LSBio, Inc.), anti- PRDX5 antibody (1:2000, Abcam), anti-GPX4 antibody (1:15,000, Abcam), anti-ACTB antibody (1:500, Abcam), and anti-GSTM5 antibody (1:5,000, Abcam). Anti- $\alpha$-tubulin mouse antibody (1:1000, Abcam) was used as the loading control for all western blots. The proteins on the membranes were detected with by an enhanced chemiluminescence (ECL) technique using ECL detection reagents.

\section{Signaling pathways}

Pathway Studio (v 9.0, Aridane Genomics, Rockville, MD, USA) program was used to predict the signaling pathways and biological functions of BPA-mediated differentially expressed ( $>2$-fold; $p<0.05$ ) proteins according to previously described procedure [29, 48]. Briefly, differentially expressed proteins were entered into the Pathway Studio in order to determine the significantly matching pathways for each protein $(p<0.05)$. To fulfill the additional objectives, groups of proteins that significantly related to particular pathways were subject to a MedScan Reader (v5.0) search, and their functional affiliation with disease processes were predicted using Pathway Studio. The signaling pathways and biological functions were confirmed by the PubMed Medline hyperlink that was embedded in each node.

\section{Statistical analysis}

The data were analyzed by one-way ANOVA using SPSS program (v. 18.0, Chicago, IL, USA), and Tukey's test was used to locate differences. $P$ values of $<0.05$ were considered statistically significant. All data are expressed as mean \pm SEM. The probabilities of the signaling pathways were determined using the Fisher's exact test $(p<0.05)$.

\section{Additional files}

Additional file 1: Table S1. Peptide sequence, matches peptides, and searching engines of identified proteins. (PDF $99 \mathrm{~kb}$ )

Additional file 2: Figure S1. Validation of the 2-DE results by western blot analysis. (A) The bars represent the densities of particular proteins bands, such as GAPDH, UQCRFS1, PRDX5, GPX4, ACTB, and GSTM5. Data are presented as mean $\pm \operatorname{SEM}(n=3)$. Values with different superscript characters $(A, B, C)$ indicate significant differences between the control and treatment samples as determined by one-way ANOVA $(P<0.05)$. (B) Representative western blot image of GAPDH, UQCRFS1, PRDX5, GPX4, ACTB, and GSTM5 probed with the respective antibodies. Figure S2. Effect of BPA on tyrosine phosphorylation status of spermatozoa. (A) Density of phosphotyrosine proteins in control and BPA-treated samples. Data are the mean of four replicates \pm SEM. Values with different upper and lower case letters $(A, B, C, a, b, c)$ are significantly different between control and treatment groups by one-way ANOVA. (B) Tyrosine-phosphorylated proteins were probed with PY 20. Lane 1: control; lane 2: $0.0001 \mu \mathrm{M}$ BPA; lane 3: $0.01 \mu \mathrm{M}$ BPA; lane 4: 1 MM BPA; and lane 5: $100 \mu \mathrm{M}$ BPA. Figure S3. Validation of separation technique of spermatozoa for proteomic analysis. Spermatozoa examined for the non-appearance of immature sperm cells and somatic cells using microscopy. (A) Differential interference contrast microscopic (DIC) image of spermatozoa used for proteomic analyses. (B) Image of hoechst staining spermatozoa. (C) Merged image. (PDF $936 \mathrm{~kb}$ )

\section{Acknowledgments}

This research was supported by a 2014 grant (14162MFDS661) from the Ministry of Food and Drug Safety of the Republic of Korea.

\section{Authors' contributions}

MSR, WSK, SJY, and YJP performed the experiments. MSR analyzed the data and performed the artwork. MSR and MGP draft the manuscript. Both MGP and BYR supervised the design of the study. All authors critically reviewed the manuscript for intellectual content and gave final approval for the version to be published.

\section{Competing interests}

The authors declare that they have no competing interests.

Received: 19 January 2016 Accepted: 28 July 2016

Published online: 09 August 2016

References

1. Calafat AM, Ye X, Wong LY, Reidy JA, Needham LL. Exposure of the U.S. population to bisphenol A and 4-tertiary-octylphenol: 2003-2004. Environ Health Perspect. 2008;16(1):39-44.

2. Rochester JR. Bisphenol A, and human health: a review of the literature. Reprod Toxicol. 2013;42:132-55.

3. Li D, Zhou Z, Qing D, He Y, Wu T, Miao M, Wang J, Weng X, Ferber JR, Herrinton L, Zhu Q, Gao E, Checkoway H, Yuan W. Occupational exposure to bisphenol-A (BPA) and the risk of self-reported male sexual dysfunction. Hum Reprod. 2010;25(2):519-27.

4. Erler C, Novak J. Bisphenol A exposure: human risk and health policy. J Pediatr Nurs. 2010;25(5):400-7.

5. Anway MD, Cupp AS, Uzumcu M, Skinner MK. Epigenetic transgenerational actions of endocrine disruptors and male fertility. Science. 2005;308(5727): 1466-9. 
6. Rahman MS, Kwon WS, Lee JS, Yoon SJ, Ryu BY, Pang MG. Bisphenol-A Affects Male Fertility via Fertility-related Proteins in Spermatozoa. Sci Rep. 2015;5:9169.

7. Heldring N, Pike A, Andersson S, Matthews J, Cheng G, Hartman J, Tujague M, Ström A, Treuter E, Warner M, Gustafsson JA. Estrogen receptors: how do they signal and what are their targets. Physiol Rev. 2007;87(3):905-31.

8. Nadal A, Ropero AB, Laribi O, Maillet M, Fuentes E, Soria B. Nongenomic actions of estrogens and xenoestrogens by binding at a plasma membrane receptor unrelated to estrogen receptor alpha and estrogen receptor beta. Proc Natl Acad Sci U S A. 2000;97(21):11603-8.

9. Ptak A, Gregoraszczuk EL. Bisphenol A induces leptin receptor expression, creating more binding sites for leptin, and activates the JAK/Stat, MAPK/ERK and PI3K/Akt signalling pathways in human ovarian cancer cell. Toxicol Lett. 2012;210(3):332-7.

10. Ptak A, Rak-Mardyła A, Gregoraszczuk EL. Cooperation of bisphenol A and leptin in inhibition of caspase-3 expression and activity in OVCAR-3 ovarian cancer cells. Toxicol In Vitro. 2013;27(6):1937-43.

11. Rago V, Giordano F, Brunelli E, Zito D, Aquila S, Carpino A. Identification of $G$ protein-coupled estrogen receptor in human and pig spermatozoa. J Anat. 2014;224(6):732-6.

12. Misao R, Niwa K, Morishita S, Fujimoto J, Nakanishi Y, Tamaya T. Immunohistochemical detection of estrogen and progesterone receptors in spermatozoa of infertile men. Int J Fertil Womens Med. 1997;42(6):421-5.

13. Park YJ, Mohamed-el SA, Kwon WS, You YA, Ryu BY, Pang MG Xenoestrogenic chemicals effectively alter sperm functional behavior in mice. Reprod Toxicol. 2011;32(4):418-24.

14. Mohamed-el SA, Park YJ, Song WH, Shin DH, You YA, Ryu BY, Pang MG. Xenoestrogenic compounds promote capacitation and an acrosome reaction in porcine sperm. Theriogenology. 2011;75(6):1161-9.

15. Benninghoff A. Toxicoproteomics-the next step in the evolution of environmental biomarkers. Toxicol Sci. 2007;95(1):1-4.

16. Wang S, Wang W, Xu Y, Tang M, Fang J, Sun H, Sun Y, Gu M, Liu Z, Zhang Z, Lin F, Wu T, Song N, Wang Z, Zhang W, Yin C. Proteomic characteristics of human sperm cryopreservation. Proteomics. 2014;14(2-3):298-310.

17. Kwon WS, Rahman MS, Lee JS, Kim J, Yoon SJ, Park YJ, You YA, Hwang S, Pang MG. A comprehensive proteomic approach to identifying capacitation related proteins in boar spermatozoa. BMC Genomics. 2014;15:897.

18. Kwon WS, Rahman MS, Lee JS, Yoon SJ, Park YJ, Pang MG. Discovery of predictive biomarkers for litter size in boar spermatozoa. Mol Cell Proteomics. 2015;14(5):1230-40.

19. Miki K, Qu W, Goulding EH, Willis WD, Bunch DO, Strader LF, Perreault SD, Eddy EM, O'Brien DA. Glyceraldehyde 3-phosphate dehydrogenase-S, a sperm-specific glycolytic enzyme, is required for sperm motility and male fertility. Proc Natl Acad Sci U S A. 2004;101(47):16501-6.

20. Gerits N, Kostenko S, Shiryaev A, Johannessen M, Moens U. Relations between the mitogen-activated protein kinase and the CAMP-dependent protein kinase pathways: comradeship and hostility. Cell Signal. 2008;20(9):1592-607.

21. Ickowicz D, Finkelstein M, Breitbart H. Mechanism of sperm capacitation and the acrosome reaction: role of protein kinases. Asian J Androl. 2012;14(6):816-21.

22. Tiwari D, Vanage G. Mutagenic effect of Bisphenol A on adult rat male germ cells and their fertility. Reprod Toxicol. 2013;40:60-8.

23. Vilela J, Hartmann A, Silva EF. Sperm impairments in adult vesper mice (Calomys laucha) caused by in utero exposure to bisphenol A. Andrologia. 2014;46(6):971-8.

24. Li X, Zhang S, Safe S. Activation of kinase pathways in MCF-7 cells by 17beta-estradiol and structurally diverse estrogenic compounds. J Steroid Biochem Mol Biol. 2006;98(2-3):122-32.

25. Zarubin T, Han J. Activation and signaling of the p38 MAP kinase pathway. Cell Res. 2005;15(1):11-8.

26. Bononi A, Agnoletto C, De Marchi E, Marchi S, Patergnani S, Bonora M Giorgi C, Missiroli S, Poletti F, Rimessi A, Pinton P. Protein kinases and phosphatases in the control of cell fate. Enzyme Res. 2011;2011:329098.

27. Rahman MB, Vandaele L, Rijsselaere T, El-Deen MS, Maes D, Shamsuddin M, Van Soom A. Bovine spermatozoa react to in vitro heat stress by activating the mitogen-activated protein kinase 14 signalling pathway. Reprod Fertil Dev. 2014;26(2):245-57

28. Rotfeld $\mathrm{H}$, Hillman P, Ickowicz D. PKA and CaMKII mediate PI3K activation in bovine sperm by inhibition of the PKC/PP1 cascade. Reproduction. 2014; 147(3):347-56.

29. Rahman MS, Kwon WS, Lee JS, Kim J, Yoon SJ, Park YJ, You YA, Hwang S, Pang MG. Sodium nitroprusside suppresses male fertility in vitro. Andrology. 2014;2(6):899-909.
30. Yoon SJ, Rahman MS, Kwon WS, Park YJ, Pang MG. Addition of Cryoprotectant Significantly Alters the Epididymal Sperm Proteome. PLoS One. 2016;11(3): e0152690

31. Højlund K, Wrzesinski K, Larsen PM, Fey SJ, Roepstorff P, Handberg A, Dela F Vinten J, McCormack JG, Reynet C, Beck-Nielsen H. Proteome analysis reveals phosphorylation of ATP synthase beta -subunit in human skeletal muscle and proteins with potential roles in type 2 diabetes. J Biol Chem. 2003;278(12):10436-42.

32. Kim SY, Lee PY, Shin HJ, Kim do H, Kang S, Moon HB, Kang SW, Kim JM, Park SG, Park BC, Yu DY, Bae KH, Lee SC. Proteomic analysis of liver tissue from HBx-transgenic mice at early stages of hepatocarcinogenesis. Proteomics. 2009;9(22):5056-66.

33. Eun SY, Woo IS, Jang HS, Jin H, Kim MY, Kim HJ, Lee JH, Chang KC, Kim JH, Seo HG. Identification of cytochrome c oxidase subunit $6 \mathrm{~A} 1$ as a suppressor of Bax-induced cell death by yeast-based functional screening. Biochem Biophys Res Commun. 2008;373(1):58-63.

34. Liao TT, Xiang Z, Zhu WB, Fan LQ. Proteome analysis of round-headed and normal spermatozoa by 2-D fluorescence difference gel electrophoresis and mass spectrometry. Asian J Androl. 2009;11(6):683-93.

35. Ghafouri-Fard S, Ousati Ashtiani Z, Sabah Golian B, Hasheminasab SM, Modarressi MH. Expression of two testis-specific genes, SPATA19 and LEMD1, in prostate cancer. Arch Med Res. 2010:41(3):195-200.

36. Temma T, Nishigori K, Onoe S, Sampei S, Kimura I, Ono M, Saji H. Radiofluorinated probe for PET imaging of fatty acid binding protein 4 in cancer. Nucl Med Biol. 2015;42(2):184-91.

37. Frayne J, Hall L. A re-evaluation of sperm protein 17 (Sp17) indicates a regulatory role in an A-kinase anchoring protein complex, rather than a unique role in sperm-zona pellucida binding. Reproduction. 2002;124(6):767-74.

38. Thompson W, Ramalho-Santos J, Sutovsky P. Ubiquitination of prohibitin in mammalian sperm mitochondria: possible roles in the regulation of mitochondrial inheritance and sperm quality control. Biol Reprod. 2003; 69(1):254-60.

39. Rashid S, Grzmil P, Drenckhahn JD, Meinhardt A, Adham I, Engel W, Neesen J. Disruption of the murine dynein light chain gene Tcte3-3 results in asthenozoospermia. Reproduction. 2010;139(1):99-111.

40. Bauer SM, Roy A, Emo J, Chapman TJ, Georas SN, Lawrence BP. The effects of maternal exposure to bisphenol $\mathrm{A}$ on allergic lung inflammation into adulthood. Toxicol Sci. 2012;130(1):82-93.

41. Wolstenholme JT, Edwards M, Shetty SR, Gatewood JD, Taylor JA, Rissman EF, Connelly JJ. Gestational exposure to bisphenol a produces transgenerational changes in behaviors and gene expression. Endocrinology. 2012;153(8):3828-38.

42. Takai $Y$, Tsutsumi O, Ikezuki Y, Hiroi H, Osuga Y, Momoeda M, Yano T, Taketani Y. Estrogen receptor-mediated effects of a xenoestrogen, bisphenol A, on preimplantation mouse embryos. Biochem Biophys Res Commun. 2000:270(3):918-21.

43. Takai $Y$, Tsutsumi O, Ikezuki Y, Kamei Y, Osuga Y, Yano T, Taketan Y. Preimplantation exposure to bisphenol A advances postnatal development Reprod Toxicol. 2001;15(1):71-4.

44. Pfeifer D, Chung YM, Hu MC. Effects of Low-Dose Bisphenol A on DNA Damage and Proliferation of Breast Cells: The Role of c-Myc. Environ Health Perspect. 2015;123(12):1271-9.

45. Yoon SJ, Kwon WS, Rahman MS, Lee JS, Pang MG. A novel approach to identifying physical markers of cryo-damage in bull spermatozoa. PLoS One. 2015;10(5):e0126232

46. Cao W, Gerton GL, Moss SB. Proteomic profiling of accessory structures from the mouse sperm flagellum. Mol Cell Proteomics. 2006;5(5):801-10.

47. Zhao C, Guo XJ, Shi ZH, Wang FQ, Huang XY, Huo R, Zhu H, Wang XR, Liu $J Y$, Zhou ZM, Sha JH. Role of translation by mitochondrial-type ribosomes during spermcapacitation: an analysis based on a proteomic approach. Proteomics. 2009:9(5):1385-99.

48. Rahman MS, Kwon WS, Karmakar PC, Yoon SJ, Ryu BY, Pang MG. Gestational Exposure to Bisphenol-A Affects the Function and Proteome Profile of F1 Spermatozoa in Adult Mice. Environ Health Perspect. 2016. http://dx.doi.org/ 10.1289/EHP378. 